\title{
From urban climate to building and material scale: a multiscale modeling approach
}

\author{
Dominique Derome $^{1}$, Aytaç Kubilay ${ }^{1,2}$, Jonas Allegrini ${ }^{1,2}$, Andrea Ferrari ${ }^{2}$, Jan Carmeliet $^{2}$ \\ ${ }^{1}$ Laboratory of Multiscale Studies in Building Physics, Empa, 8600 Dübendorf, Switzerland \\ ${ }^{2}$ Chair of Building Physics, ETH Zurich, 8092 Zürich, Switzerland
}

\begin{abstract}
Modeling the local urban climate is a natural extension of the field of building simulation. To achieve appropriate modeling of the urban climate, a multiscale approach is proposed. Several models at different scales, such as city, neighborhood and street canyon, building and material scale, allow predicting, analyzing and mitigating negative effects of local heat islands on urban thermal comfort and building cooling demand.
\end{abstract}

\section{Introduction}

Our cities are nowadays confronted with urban heat islands (UHI), showing that average air temperature can be considerably higher in cities compared to the surrounding rural environment, especially at night. Additionally, cities are threatened with local heat islands (LHI), where certain areas show locally much higher temperatures compared to their surrounding neighborhoods.

Growing evidence indicated that heat waves are more frequent under increased greenhouse forcing (e.g. Seneviratne et al. 2012, Hartmann et al. 2013, Schleussner et al. 2017). Climate simulations predict an increase of the number and intensity of heat waves and hot extremes in many regions in the world (Sillmann et al. 2013, Fischer et al. 2014) even for the more moderate scenarios of global warming of $1.5^{\circ} \mathrm{C}$ or $2^{\circ} \mathrm{C}$ (Seneviratne et al. 2016, Wartenburger et al. 2017, Dosio et al. 2018). In future, a threat is that heat waves associated with higher daytime temperatures and reduced nighttime cooling might exceed the limits of thermoregulation of the human body and affect human health. In addition to UHI leading to a reduction of urban thermal comfort, an increase in building energy demand, especially in space cooling demand during warm periods is foreseen. Given local and global climate change and growth of world's population and economy, the world global space cooling demand for buildings is expected to strongly increase in future. In 2010 the global cooling consumption from the residential sector represented $4.4 \%$ of the energy required for the total space conditioning of buildings. The share due to cooling is expected to increase to $35 \%$ in 2050 and $61 \%$ in 2100 (Santamouris 2016, Santamouris et al 2016, Isaac and van Vuuren 2009).
Modeling the local urban climate is a natural extension of the field of building simulation. To properly capture and understand local street conditions, wind flow either forced or buoyancy driven, heat and mass exchanges of the air with porous media including vegetation, solar radiation and radiative exchanges have all to be adequately modeled. A most challenging aspect of climatic loading, wetting by rain deposition, should also be included.

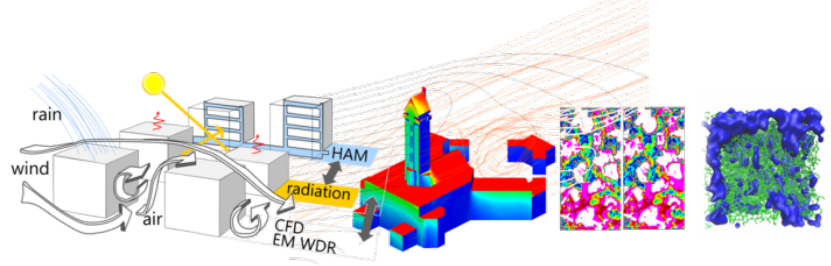

Figure 1: Representation of the different scales needed: urban context, building and porous materials from pores to nanostructure.

In this conference presentation, several models at different scales, as schematized in Figure 1, from city to material scales, with an emphasizing on street canyon, are briefly discussed in order to allow predicting and analyzing local heat islands, in an effort towards mitigating their negative effects on urban thermal comfort and building cooling demand.

\section{Multiscale modeling \\ Interactions of regional and urban climate}

The development of urban canopy parameterizations in mesoscale models enables a better representation of the mesoscale forcing of urban weather and climate. Numerical weather prediction models at sub-kilometer resolution can be used to simulate the urban heat island (UHI) (order of $0.1-1 \mathrm{~km}$ grid cells) while effects of buildings are parametrized. As an example, the urban heat island effect in Zurich was simulated with the climate version of COSMO using the urban parametrization model DCEP in order to capture the local atmospheric circulation influenced by the orography and the presence of Lake Zurich (Mussetti et al. 2016).

The results of the meteorological model are coupled with an urban climate model based on computational fluid dynamics (CFD) (OpenFOAM) at city and neighborhood 
scale via a one-way nesting method. This CFD based urban climate model in contrast to mesoscale models, also resolves the physical phenomena at street canyon scale (Vonlanthen et al. 2017).

\section{Urban to building scales: the need for building- resolved analysis}

It becomes clear that models need to take into account accurately the building and street geometry, meaning buildings are geometrically resolved in CFD. Also needed is to take into account aspects such as the different types of urban materials and the presence of vegetation, the particular weather conditions (wind, sunshine, rain, temperature, relative humidity), shadowing, as well as the local urban water cycle. A schematic of the model that shows the coupled physical processes is given in Figure 2.

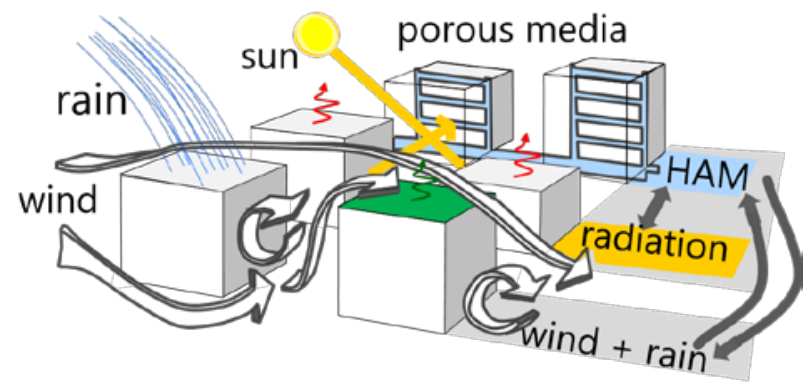

HAM heat and moisture transport in porous materials (building materials pavements, soils, ...) including phase change: evaporative cooling CFD air flow due to wind and buoyancy

WDR wind driven rain, Eulerian multi-phase model

Radiation short and longwave radiation using view-factor method

Figure 2: Schematic representation of couplings in metamodel for local urban climate.

As an example of the required level of information a vegetation model is described here, which models the impact of vegetation on momentum, heat, moisture, radiation and the water cycle in an integrated approach, for plants such as trees and planted areas, e.g. grass, via a porous medium approach. Vegetation intercepts solar radiation, providing shading to the ground, and offers natural cooling through transpiration, cooling the air. However, vegetation also slows down the air flow and its transpirative cooling potential is strongly dependent on the water availability in the soil. The tree or the grass is modeled as porous medium, including source/sink terms for heat, mass and momentum fluxes. A radiation model is developed to model the short-wave and longwave radiative heat fluxes between the leaf surfaces and the surrounding environment as well as the extinction of radiation passing through the porous medium. A finite volume approach is applied for the discretization of the vegetation foliage where the leaves of the tree are aggregated into finite volumes. The heat and mass exchanges are determined from a leaf energy balance model applied for each leaf in the discrete volume. This enables a description of the heat and mass fluxes from vegetation with a realistic geometry and leaf density distribution. Furthermore, the transpiration process at the leaf surfaces can be coupled to the water availability at the roots of the plants (Manickathan et al 2018).

The proposed approach allows analyzing the urban climate at street canyon scale evaluating the different contributions of convective cooling, sensible heat transfer due to rain, evaporative cooling, thermal storage throughout the day, in addition to shadowing and transpirative cooling by vegetation to the urban thermal comfort at street canyon scale (Kubilay et al. 2018 a, b).

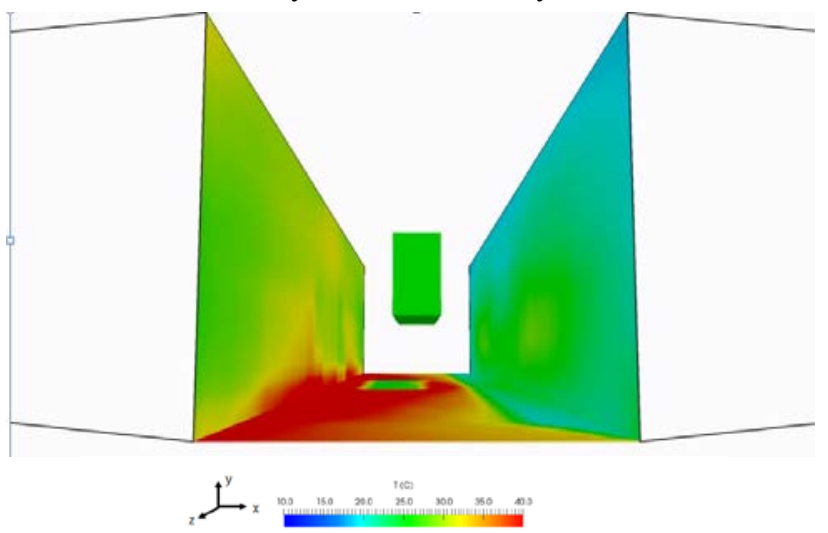

Figure 3: Example of results of surface temperature in the street canyon with a row of trees.

As an example of further analysis, the results of the urban climate model allow to consider the influence of urban parameters on thermal comfort by determining the variables at a certain location in the street canyon, such as air temperature, air speed, radiant temperature and relative humidity, all taken into account with proper couplings, as shown in Figure 3 where surface temperatures are the results of all the heat and mass transport exchanges. These variables can yield the determination of the thermal comfort/heat stress using a common thermal comfort indicator, such as the UTCI (Universal Thermal Climate Index, Bröde et al., 2018). In Figure 4, the influence of a tree on the UTCI for a person (red box) standing below a tree (green box) are displayed, showing the positive effect of a tree mainly through shadowing on the thermal comfort.

For buildings in urban environments, the space cooling demand is strongly influenced by the local urban climate and can be quite different compared to buildings in rural areas. The shortwave radiation entering the street canyons is entrapped between the buildings due to multiple reflections between street canyon surfaces. Additionally, sunlit surfaces exchange longwave radiation with non-sunlit surfaces and heat them up. These increased surface temperatures have a direct impact on the energy performance of the buildings and on the local air temperatures. Another important impact is the increased local air temperatures due to the urban heat island effect. 


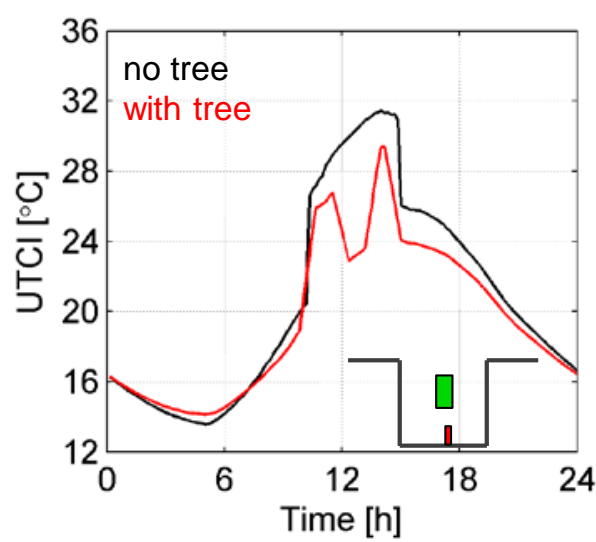

Figure 4: Example of results from the modeling framework presented in Figure 1, for the thermal comfort conditions of someone standing under a tree in a street subjected to light wind and sunny conditions in summer.

\section{Integrating the material scale}

The coupled heat-moisture transport model (indicated as HAM in Figure 2 allows for the improvement in thermal comfort as a result of evaporation from wetted urban surfaces. Figure 5 shows the decrease in the street surface temperatures and the resulting reduction in UTCI for someone standing in a street canyon. The street surface, which is composed of brick pavement, is wetted during the initial 10 hours at different intensities. The reduction in temperatures depends on various parameters, such as moisture availability near the surface and material liquid capacity and permeability.
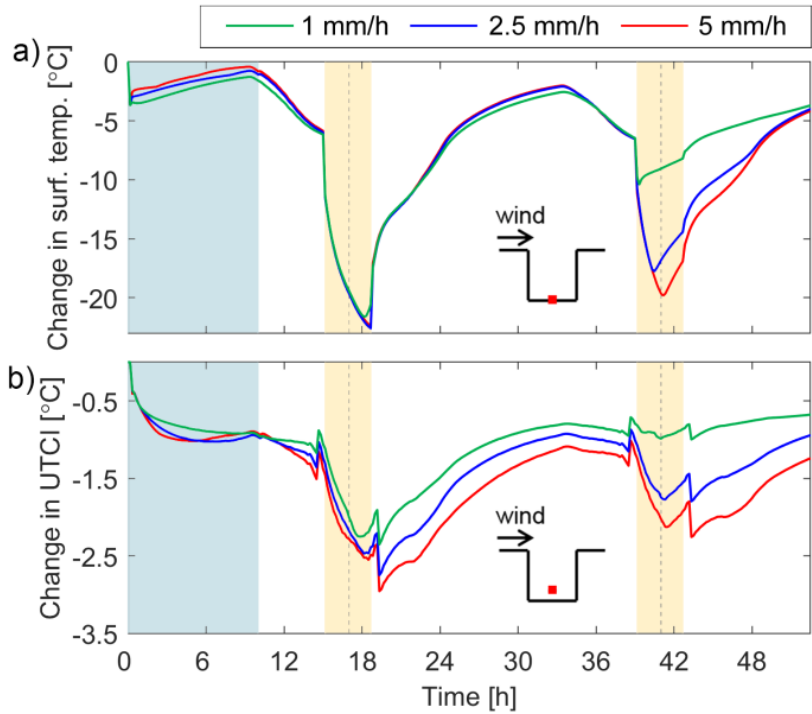

Figure 5: Temporal variation of reduction in a) surface temperature of wetted brick pavement and b) UTCI for someone standing in a street canyon. Blue denotes the time interval of wetting and yellow denotes direct solar radiation at street surface.

The model can further be used in the design of new urban materials, which can help in the mitigation of local heat islands. With respect to evaporative cooling, there is a strong need to optimize water retention of urban materials by tailoring their pore structure. This requires the use of pore-scale simulation of two-phase flow in porous media and upscaling using pore-network models for the determination of the macroscopic fluid transport properties.

As an example, it was recently shown that hydrophobic macro-porous materials, such as porous asphalt with pore sizes ranging from micrometer to millimeter size, can retain water in their pore structures long after having been wetted by rain or by artificial wetting (spraying or through dedicated water piping systems), as such opening potentials for new evaporative cooling materials (Lal 2016). To study the two-phase flow in such complex materials, several models were developed using multiphase lattice Boltzmann model (PM-LBM). The use of LBM was motivated by the need for the explicit tracking of the complex liquid-vapor interface during gravity-driven drainage in porous asphalt. Such approach is also used to study drying processes. To predict the unsaturated permeability of building materials, porenetwork modeling has been used (Carmeliet et al. 1999, 2004, Vandersteen et al. 2003) and is under continuing development. The understanding and information obtained from pore-scale investigations enables to understand macro-scale transport processes, and to explore the potential of new evaporative cooling materials, via an efficient computational mean.

\section{Conclusion}

The multiscale urban climate model is presently further developed and actively used to understand the impact of different urban parameters on the local heat island effect and towards proposing adequate mitigation measures. The approach allows investigation of multiple aspects such as studying the impact of (1) radiation properties of building surfaces (albedo value), (2) the heat-moisture transport and capacity properties of building porous materials, (3) the presence of vegetation such as green roofs and facades, trees and squares, (4) the role of impervious versus porous substrates (different pavements and sublayers, different soils, cover ratio), (5) the presence of urban shadowing (often via nonpermanent devices), (6) the role of active evaporative cooling by water spraying during heat waves using, for example, collected urban water.

\section{Acknowledgement}

This research project is supported by the Swiss Competence Center for Energy Research SCCER FEEB\&D of the Swiss Innovation Agency lnnosuisse.

\section{References}

Bröde, P., Fiala, D., Lemke, B., Kjellström, T. (2018). Estimated work ability in warm outdoor environments depends on the chosen heat stress assessment met-ric, Int. J., Biometeorol., 62 (3), 331345.

Carmeliet J., Descamps F, Houvenaghel G: Multiscale network model for simulating liquid water and water 
vapour transfer properties of porous materials. Transport in Porous Media 35: 67-88, 1999

Carmeliet J, Delerue J.F, Vandersteen K, Roels S. (2004) Three-dimensional liquid transport in concrete cracks. International Journal for Numerical and Analytical Methods in Geomechanics 28: 671-687

Dosio, A., L. Mentaschi, E.M. Fischer, and K. Wyser, (2018). Extreme heat waves under $1.5^{\circ} \mathrm{C}$ and $2{ }^{\circ} \mathrm{C}$ global warming, Environ. Res. Lett. 13054006.

Fischer, E.M., Sedláček, J., Hawkins, E., and Knutti, R. (2014). Models agree on forced response pattern of precipitation and temperature extremes, Geophys. Res. Lett., 41.

Hartmann, D.L., A.M.G. Klein Tank, M. Rusticucci, L.V. Alexander, S. Br.nnimann, Y. Charabi, F.J. Dentener, E.J. Dlugokencky, D.R. Easterling, A. Kaplan, B.J. Soden, P.W. Thorne, M. Wild and P.M. Zhai. (2013): Observations: Atmosphere and Surface. In: Climate Change 2013: The Physical Science Basis. Contribution of Working Group I to the Fifth Assessment Report of the Intergovernmental Panel on Climate Change [Stocker, T.F., D. Qin, G.-K. Plattner, M. Tignor, S.K. Allen, J. Boschung, A. Nauels, Y. Xia, V. Bex and P.M. Midgley (eds.)]. Cambridge University Press, Cambridge, United Kingdom and New York, NY, USA.

Isaac M, van Vuuren DP. (2009). Modeling global residential sector energy demand for heating and air conditioning in the context of climate change. Energy Policy 37, 507-521.

Kubilay, A., Derome, D. and J. Carmeliet (2018a). Coupling of physical phenomena in urban microclimate: A model integrating air flow, winddriven rain, radiation and transport in building materials. Urban Climate 24, 398-418.

Kubilay, A., Carmeliet, J. and D. Derome (2018b). Using rain and vegetation to improve thermal comfort in a hot street canyon with fully-integrated urban climate modeling. Proceedings from the $7^{\text {th }}$ International Building Physics Conference. Syracuse, NY (USA), 23-26 September.

Lal S. (2016) Multiscale investigation and numerical modelling of imbibition, drainage and drying of macroporous medium, PhD thesis ETH Zurich No. 23628.

Manickathan, L., Defraeye, T., Allegrini, J., Derome, D. and J. Carmeliet (2018). Parametric study of the influence of environmental factors and tree properties on the transpirative cooling effect of trees. Agr. Forest Meteorol. 248, 259-274.
Mussetti G, Brunner D, Henne S, Allegrini J, Wouters H, Schubert S, Carmeliet J. 2016. Impact of model resolution and urban parameterization on urban climate simulation: a case study for Zürich. COSMO/CLM/ART User Seminar 2016. 7-9 March 2016, Offenbach.

Santamouris M; Kolokotsa D. (2016). 'Passive cooling of buildings: Present and future needs: Recent progress on passive cooling convective technologies', Advanced Environmental Wind Engineering, pp. 75 88, 10.1007/978-4-431-55912-2_4.

Santamouris M. (2016). Cooling the buildings - past, present and future. Energy and Buildings 128, 617638.

Schleussner, C.F., P. Pfleiderer, and E.M. Fischer. (2017): In the observational record half a degree matters. Nature Climate Change, 7, 460-462.

Seneviratne, S.I., N. Nicholls, D. Easterling, C.M. Goodess, S. Kanae, J. Kossin, Y. Luo, J. Marengo, K. McInnes, M. Rahimi, M. Reichstein, A. Sorteberg, C. Vera, and X. Zhang. (2012): Changes in climate extremes and their impacts on the natural physical environment. In: Managing the Risks of Extreme Events and Disasters to Advance Climate Change Adaptation[Field, C.B., V. Barros, T.F. Stocker, D. Qin, D.J. Dokken, K.L. Ebi, M.D. Mastrandrea, K.J. Mach, G.-K. Plattner, S.K. Allen, M. Tignor, and P.M. Midgley (eds.)]. A Special Report of Working Groups I and II of the Intergovernmental Panel on Climate Change, pp. 109-230.

Sillmann, J.,V.V.Kharin, F. W. Zwiers,X. Zhang, and D. Bronaugh (2013). Climate extremes indices in the CMIP5 multimodel ensemble: Part 2. Future climate projections, J. Geophys. Res. Atmos., 118, 24732493, doi:10.1002/jgrd.50188.

Vandersteen K, Carmeliet J, Feyen J. (2003) A network approach to derive unsaturated hydraulic properties of a rough-walled fracture. Transport in Porous Media 50(3): 197-221

Vonlanthen M., Allegrini J., Carmeliet J. (2017) Multiscale interaction between a cluster of buildings and the ABL de-veloping over a real terrain, Urban Climate, 20:1-19.

Wartenburger, R., Hirschi, M., Donat, M.G., Greve, P., Pitman, A.J. and Seneviratne, S.I. (2017): Changes in regional climate extremes as a function of global mean temperature: an interactive plotting framework. Geosci. Model Dev., 10, 3609-3634 